\title{
Experimental validation for the compensation method of nonlinearities in periodic magnets
}

\author{
Simona Bettoni* \\ CERN, CH-1211 Geneva 23, Switzerland and Laboratori Nazionali di Frascati INFN-LNF, \\ Via E. Fermi, 40, 00044 Frascati (Roma), Italy \\ Susanna Guiducci, Miro Preger, and Pantaleo Raimondi ${ }^{\dagger}$ \\ Laboratori Nazionali di Frascati INFN-LNF, Via E. Fermi, 40, 00044 Frascati (Roma), Italy
}

(Received 3 March 2014; published 16 October 2014)

\begin{abstract}
Nonlinearities from any periodic magnet in accelerators may strongly degrade the dynamics of the beams. This may be especially critical for magnets where the beam excursions are comparable to the good field region, as for example in wigglers, since the beam trajectory can be of the order of the pole width. A general method based on alternately shifting the magnetic axis of each pole to compensate this effect was proposed and applied to the DA $\Phi$ NE wigglers, where an important integrated octupole was measured. This approach has been optimized by multipolar analyses and the effect on the beam dynamics verified by tracking studies. In this paper we report about the experimental validation of the magnetic model and the verification of the method by beam based measurements. The latter were performed after all the wigglers in the DA $\Phi N E$ main rings had been modified according to the optimal configuration. These measurements were in agreement with the expectations and allowed experimentally proving the method.
\end{abstract}

DOI: 10.1103/PhysRevSTAB.17.102401

PACS numbers: 29.27.-a, 29.20.-c

\section{INTRODUCTION}

Wigglers are normally installed in synchrotron light source machines, damping rings and particle factories to increase the radiation damping and control the emittance. In particular in the damping rings of the International Linear Collider (ILC) [1] and Compact Linear Collider (CLIC) [2] a large number of wigglers is installed for these reasons. The large excursion of the beam trajectory with respect to the magnet axis of the wiggler, combined with the field roll-off, can be an important source of nonlinearity for the beams. The largest nonlinear contributions arise from the integrated odd multipoles of the field expansion around the beam trajectory in U.S. notation $(n=0$ for dipole, $n=1$ for quadrupole, ...), which add up for each pole, whereas the even ones vanish for the periodicity of the magnet. The increase of the pole width to improve the transverse field uniformity is normally unpractical or impossible if a high peak field has to be generated and in any case it reduces the maximum achievable field in the

\footnotetext{
*Present address: Paul Scherrer Institut (PSI), 5232 Villigen, Switzerland.

simona.bettoni@psi.ch

'Present address: European Synchrotron Radiation Facility (ESRF), 38043 Grenoble, Cedex 9, France.
}

Published by the American Physical Society under the terms of the Creative Commons Attribution 3.0 License. Further distribution of this work must maintain attribution to the author(s) and the published article's title, journal citation, and DOI. midplane. Several possibilities have been explored in the past to mitigate the nonlinearities: pole shims can be added [3] or jaws can be installed at the extremities of the magnet [4]. In the first case the integral is reduced only in the pole region, so that this method is not convenient for short poles wigglers. The second approach may be useful only if one specific order has to be compensated and if the correction is small enough that the other orders are not significantly distorted by the jaw. This technique is not optimal for long wigglers then, because it corrects the nonlinearities only at the end of the device. A novel approach, capable of compensating a specific odd component of the field integrals without affecting the even terms, was proposed and extensively described in [5]. A brief summary of the concept, focusing on the implemented modifications, is given in Sec. II. The application and the optimization on the wigglers installed in the DA $\Phi N E$ main rings are described in Sec. III. A validation of the 3D modeling by $2 \mathrm{D}$ magnetic field map measurements in the midplane is described in Sec. III B. After this verification all the wigglers in the DA $\Phi N E$ main rings were modified according to the optimal computed configuration. The method was finally verified by beam based measurements reported in Sec. III C.

\section{THE METHOD}

The proposed approach consists in disentangling the magnetic and geometric axes, in such a way that in each semiperiod of the magnet the beam trajectory changes sign 
likewise. By opportunely choosing the shift of the magnetic axis, it is possible to vanish the integral of the multipolar component of the field with respect to the beam reference trajectory in each semiperiod of the magnet.

The method, extensively described in [5], is only briefly recalled here. Let $(x, y, z)$ be a Cartesian coordinate system, with $z$ the longitudinal, $x$ the horizontal and $y$ the vertical dimension respectively. Defining $x_{T}$ the beam reference trajectory in the midplane $(x, 0, z)$ and $\tilde{x}$ the horizontal coordinate with respect to $x_{T}$ in this plane, the Taylor expansion of the vertical component of the magnetic field around $x_{T}$ can be written as

$$
\begin{aligned}
B_{y}(\tilde{x}, 0, z) & =\left.\sum_{n=0}^{\infty} \frac{1}{n !} \frac{\partial^{n} B_{y}(\tilde{x}, 0, z)}{\partial \tilde{x}^{n}} \cdot \tilde{x}^{n}\right|_{\tilde{x}=0} \\
& \equiv \sum_{n=0}^{\infty} b_{n}^{T} \tilde{x}^{n}
\end{aligned}
$$

where the derivatives are calculated along the beam trajectory. The coefficients $b_{n}{ }^{T}$ correspond to the multipoles with respect to the beam trajectory $\left(b_{0}{ }^{T}\right.$ to the dipole, $b_{1}{ }^{T}$ to the quadrupole, ...). These terms can be written as a function of the coefficients of the field expansion around the geometric axis $b_{k}{ }^{A}$ :

$$
b_{n}^{T}=\sum_{k=n}^{\infty} c_{k} \cdot b_{k}^{A} \cdot x_{T}^{k-n},
$$

where $c_{k}$ are positive coefficients resulting from the Taylor expansion of the field. To first approximation the effect experienced by the beam is given by the integral of each $b_{n}^{T}$, $I_{n}$, defined as

$$
I_{n} \equiv \int_{-\infty}^{+\infty} b_{n}^{T} \cdot d s
$$

where $s$ is the curvilinear longitudinal coordinate of the reference trajectory.

In conventional wigglers (symmetric in the $x-y$ plane with respect to the $y$ axis) the odd terms in the field expansion around the axis $b_{2 k+1}^{A}$ vanish for symmetry. Therefore the integrals of the multipole coefficients with respect to the trajectory $b_{n}^{T}$ are determined by the even terms of the on axis expansion $b_{2 k}^{A}$. In particular even terms with respect to the trajectory can be written as

$$
b_{2 n}^{T}=c_{2 k} \cdot b_{2 k}^{A}+c_{2 k+2} \cdot b_{2 k+2}^{A} \cdot x_{T}^{2}+\cdots .
$$

The integrals of the even terms $b_{2 k}^{A}$, which are multiplied by even powers of $x_{T}$, are canceled in each period of the magnet, because of the alternating sign of the field. As a consequence the even integrals $I_{2 n}$ vanish in any period of the device. The integrals of the odd terms $b_{2 n+1}^{T}$ are determined by even terms with respect to the on axis terms multiplied by odd powers of the trajectory:

$$
b_{2 n+1}^{T}=c_{2 n+2} \cdot b_{2 n+2}^{A} \cdot x_{T}+c_{2 j+2} \cdot b_{2 j+4}^{A} \cdot x_{T}^{3}+\cdots
$$

so they do not cancel and the multipole components in Eq. (5) sum up over all the periods.

This method is general and can be applied to any periodic magnet. It has been optimized to the DA $\Phi$ NE main rings wigglers, and in this particular case simulations indicated that the integrated octupole can be compensated and all the other odd integrals reduced.

\section{THE APPLICATION TO DA $\Phi$ NE}

Four normal conducting wigglers per ring are installed in the $\Phi$ factory DA $\Phi$ NE to reduce the damping time. The most important parameters of these devices are listed in Table I.

In DA $\Phi N E$ electrons and positrons oscillate along a quasisinusoidal trajectory with an amplitude of nearly $1.3 \mathrm{~cm}$ in the wigglers, because of the relatively low beam energy $(510 \mathrm{MeV})$ and the large magnetic field of the device. Due to such a beam excursion the DA $\Phi N E$ wigglers were a source of strong nonlinearities for the machine. In particular, a large integrated octupole with respect to the beam trajectory was evidenced by tune shift measurements as a function of the trajectory amplitude in the wigglers [6]. This nonlinearity limited the dynamic aperture and the beam lifetime, dominated by the Touschek effect.

Several approaches have been tried in the past to mitigate this effect in the DA $\Phi N E$ wigglers, the most effective being pole shimming to minimize the field roll-off. This intervention decreased the integrated octupole by about a factor 2 , but it was not really decisive, having also the drawback of reducing the peak field by $15 \%$ because of the gap increase [7]. In the following this configuration will be taken as the reference for comparison with the results of the method described in [5].

The proposed approach consists in shifting the magnetic axis to change the sign of the beam trajectory in each semiperiod of the wiggler and by this canceling the integrals of the terms in Eq. (5). Several options can be considered to obtain such a result: apply pole shims, cut the poles or shift them. The first option was rejected because of the reasons described above. The second possibility has been already explored and described in detail in [5]. The drawback of this approach is the pole width reduction, which makes the iron saturate at lower current, requiring pole shims (although thinner than that described in [7]) to

TABLE I. Main parameters of the DA $\Phi$ NE wigglers.

\begin{tabular}{lccc}
\hline \hline & Central poles & Terminal poles \\
\hline Number of poles & 5 & & 2 \\
Nominal current (A) & 693 & & 564 \\
Peak field (T) & 1.75 & 1.45 \\
Pole width (cm) & & 64 & \\
Period (cm) & & 6.7 & \\
Gap $(\mathrm{cm})$ & & 3.7 & \\
\hline \hline
\end{tabular}




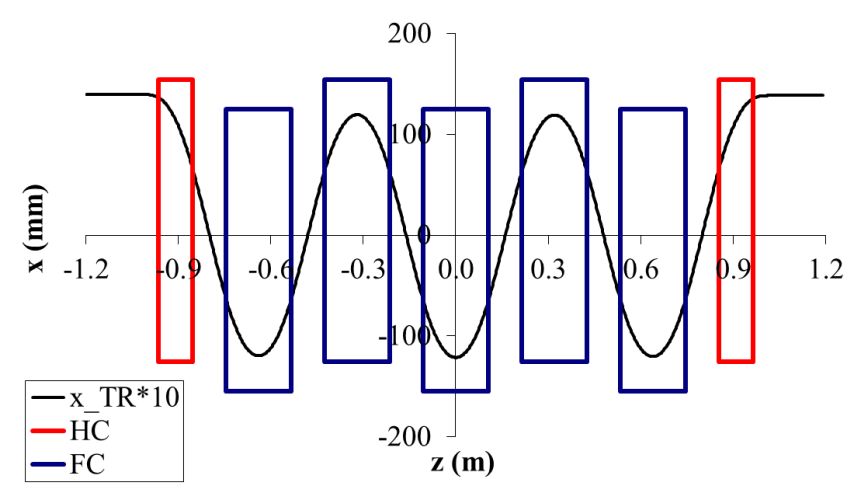

FIG. 1. Schematic top view of the wiggler. The terminal (HC) and the central (FC) coils are indicated in red and in blue respectively. The reference trajectory $x_{T}$ is also indicated in black.

then reduce the dependence of the integrated multipoles on the beam-wiggler misalignment [8]. In the following we concentrate on the third possibility, which consists instead in alternatively displacing each pole in the direction of the beam reference trajectory to find the optimal shift to vanish the integral $I_{3}$, as schematically shown in Fig. 1.

This solution, oppositely to the shimming option, increases the good field region by reducing the iron poles saturation (less current is necessary to produce the same magnetic field in the midplane). This approach can be very easily applied to any periodic magnet without additional cost during the design phase. In practice for the DA $\Phi N E$ wigglers it was necessary just to dismount the lower part of the poles, remove the previously installed shims and reinstall the shifted poles. By applying this modification the gap was reduced and the field in the midplane consequently increased. In particular, in this new configuration the peak field at $550 \mathrm{~A}$ in the main coils is slightly larger than the one at the previously nominal $693 \mathrm{~A}$. In parallel to this intervention, a new connection scheme of the terminal coils has also been optimized and implemented [9]. These modifications gave more than $30 \%$ reduction in the electrical power consumption.

\section{A. The optimization}

The field integrals $I_{n}$ as a function of the magnetic axis shift have been computed from 3D magnetic models of the DA $\Phi N E$ wigglers done in TosCA [10]. From these models the vertical component of the field was computed in points laying on segments orthogonal to the reference trajectory at several $s$ along the beam reference trajectory in the midplane of the wiggler. Then, to calculate the coefficients $b_{n}^{T}$, a fourth-order polynomial fit was applied to these values. The integrals of the $b_{n}^{T}$ along the total wiggler length for the optimal configuration are reported in Table II. The integral $I_{3}$ is a linear function of the magnetic axis offset up to more than $1 \mathrm{~cm}$ shift of the magnetic axis and in particular the integral vanishes for $\pm 7.3 \mathrm{~mm}$ axis displacement [8], as shown in Fig. 2.
TABLE II. Simulated integrated $b_{n}^{T}$ for the aligned and for the $\pm 7.3 \mathrm{~mm}$ shifted poles configuration. The units of $I_{n}$ are $T \cdot m^{1-n}$. The increase of the integrated second order is given by the peak field increase due to the removal of the shims previously installed to improve the transverse field uniformity. We did not apply any correction to this, because this goes to the right direction for the DA $\Phi N E$ main rings dynamic aperture maximization [11]. As expected, the integral of the quadrupolar term (odd order) is reduced.

\begin{tabular}{lcc}
\hline \hline & Aligned poles & Shifted poles \\
\hline$I_{0}$ & 0.00 & 0.00 \\
$I_{1}$ & 2.58 & 2.10 \\
$I_{2}$ & -1.10 & -1.38 \\
$I_{3}$ & 279.61 & 0.07 \\
\hline \hline
\end{tabular}

Simulations show that the integrated third order coefficient can be suppressed to below the model precision by optimizing the axis shift. As expected from Eq. (5), $b_{3}^{T}$ changes sign in each semiperiod of the wiggler and the contributions to $I_{3}$ in the poles region cancel the ones in between, as shown in Fig. 3.

Polynomial coefficients of the vertical component of the magnetic field were used to quantify the nonlinearities, since they could not otherwise be calculated along a circumference, where the beam excursion is larger than the half gap. Also tracking studies were therefore performed to verify this approach. In particular test particles entering in the wiggler field with trajectories parallel to $x_{T}$ at several horizontal offsets $\Delta x$ were simulated and the angle $x_{E}^{\prime}$ and the position $x_{E}$ at the exit of the magnet were determined. The calculations were performed by computing the first and the second integral of the field along the longitudinal axis and by using the tracking ToscA postprocessor routine [10] as well.

The results of the tracking studies are shown in Fig. 4, where the position and angle at the exit of the DA $\Phi$ NE wiggler, computed using the simulated field map as a function of the $x$ offset at the entrance $\Delta x$, are reported.

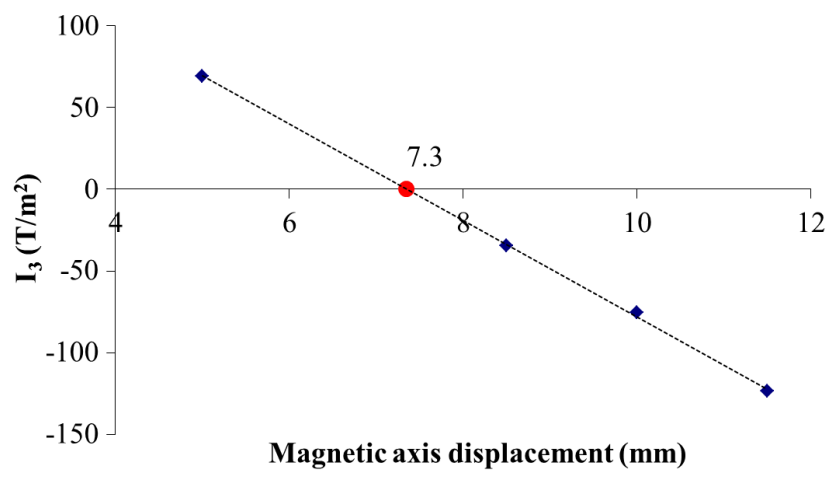

FIG. 2. Simulated $I_{3}^{T}$ as a function of the shift of the magnetic axis position. 


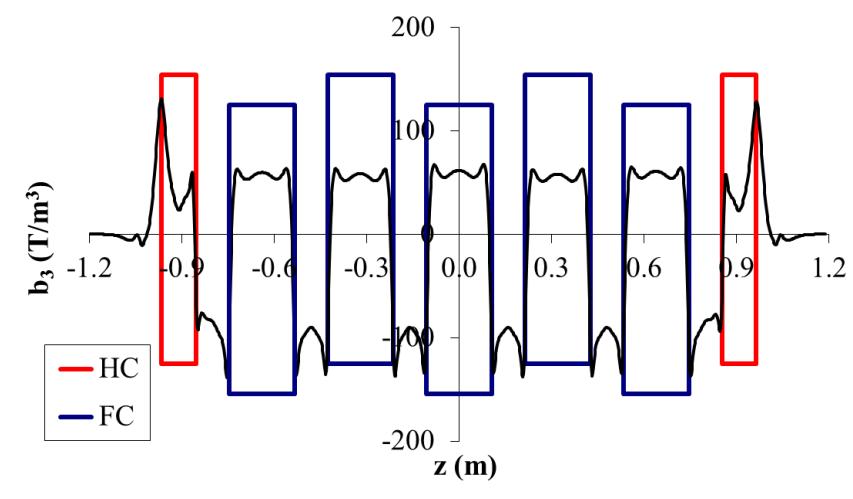

FIG. 3. Simulated $b_{3}^{T}$ along the longitudinal position for the optimal case of $\pm 7.3 \mathrm{~mm}$ axis shift.

The cubic dependence of the exit angle computed using the field map generated by the wiggler with the aligned poles was strongly reduced in the range of $\pm 4 \mathrm{~cm}$ around the beam reference trajectory. The exit angle can be decomposed to a polynomial as

$$
x_{E}^{\prime} \equiv \sum_{k=0}^{\infty} a_{k} \cdot \Delta x^{k}
$$

where each of the $a_{k}$ terms corresponds to an integrated multipolar order. The strong reduction of the third order term confirms the results of the field expansion and the strong impact of the modification on the beam dynamics.

\section{B. The magnetic measurements}

Before applying this correction to all the wigglers installed in DA $\Phi N E$, a spare magnet was modified to verify the magnetic model by comparing the measured field map in the midplane with the simulations expectations. In Fig. 5 the top view of the wiggler during the modification is shown.

The vertical component of the magnetic field in the midplane was measured by two Hall probes mounted on a

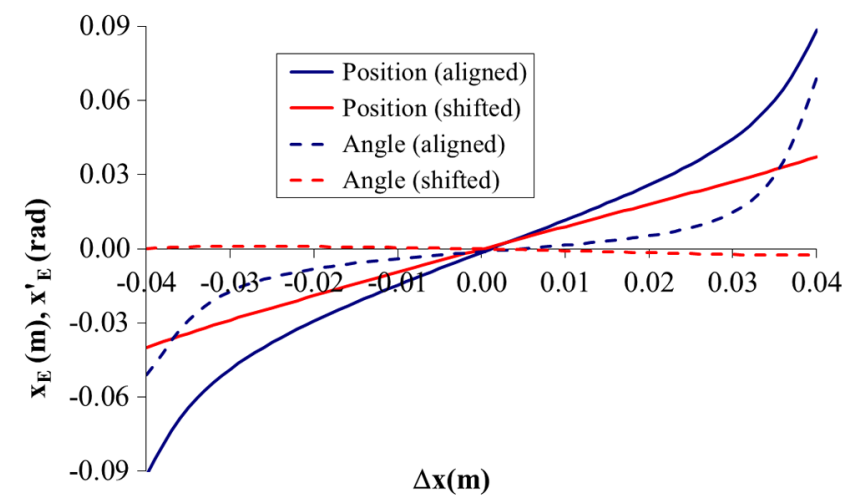

FIG. 4. Simulated exit angle and position as a function of the entrance shift $\Delta x$ with respect to the reference trajectory before (aligned) and after (shifted) the modification.

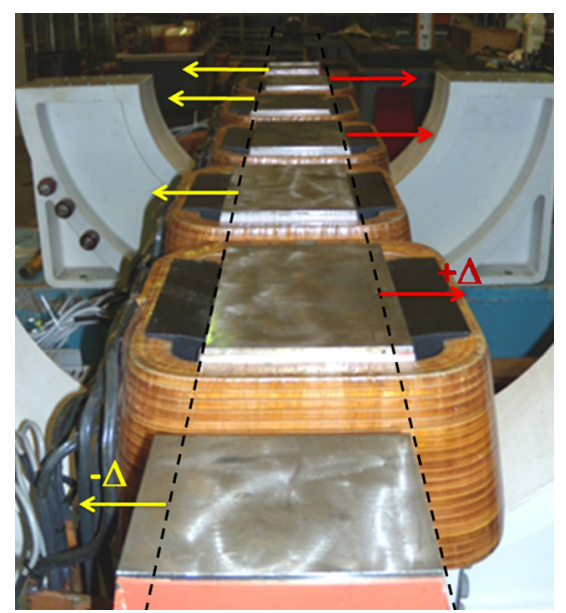

FIG. 5. Top view of the bottom half of the spare wiggler during the modification.

single remotely controlled support. The probes were moved in the midplane transversally in the range of $\pm 7 \mathrm{~cm}$ with $10 \mathrm{~mm}$ step and longitudinally in the range of $\pm 1.35 \mathrm{~m}$ with $8.35 \mathrm{~mm}$ step. The measurements have been performed at the excitation current of $550 \mathrm{~A}$ (operational value for the Siddharta experiment [3]), and also at 500 and $450 \mathrm{~A}$ in view of a possible further electrical consumption reduction for the following KLOE-2 run [12]. Here only the 550 A case is discussed. Details about the other configurations can be found in [13].

The comparison of the vertical component of the magnetic field measured in the midplane at the center of the wiggler and the predictions of the magnetic model are shown in Fig. 6. The maximum discrepancy was about $0.2 \%$, which corresponds to the stability of the power supplies during the measurements [9]. The coefficients of the field expansion around the reference trajectory $b_{n}^{T}$ higher than the dipolar order are shown in Fig. 7 for the central period of the device. The agreement was sufficient to confirm the results of the 3D magnetic modeling and, consequently, the multipolar and tracking studies.

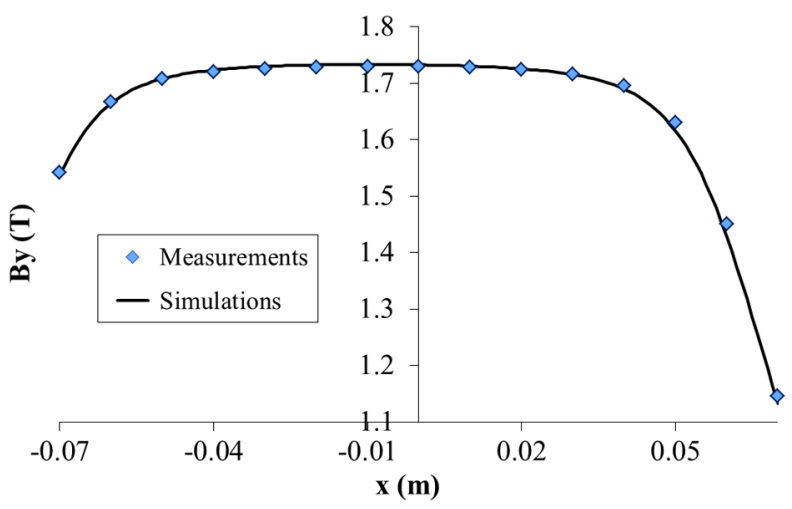

FIG. 6. Measured vertical component of the magnetic field of the modified wiggler compared to the simulations at the center of the wiggler in the midplane. 


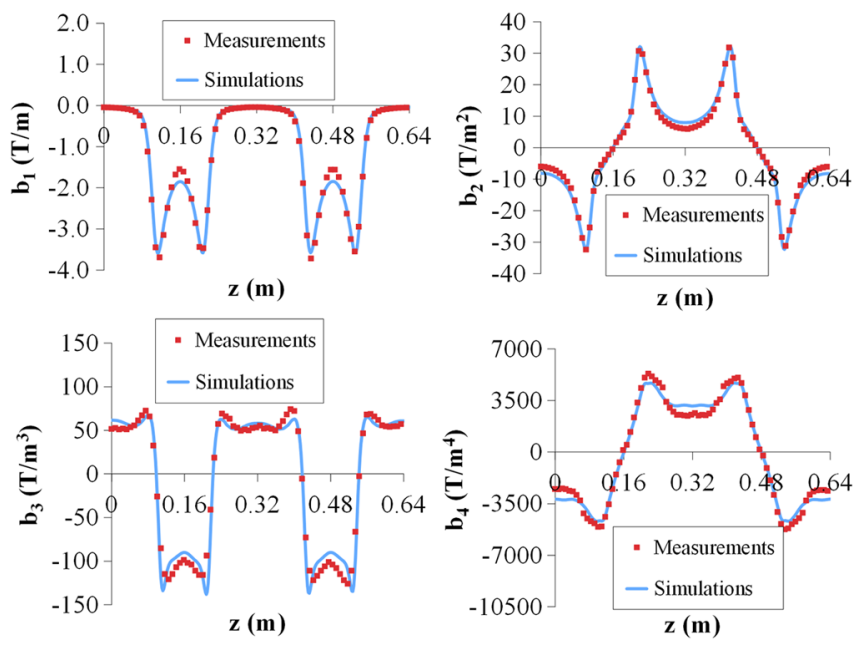

FIG. 7. Coefficients of the field expansion calculated from the measured field map on the spare wiggler after the modification and comparison with the simulation expectations (fourth order polynomial fit applied in a range of $\pm 4 \mathrm{~cm}$ around the reference beam trajectory).

After this successful result, all the wigglers in the $D A \Phi N E$ main rings were modified according to the optimized configuration. For these wigglers the shift was changed to $\pm 8 \mathrm{~mm}$ instead of $\pm 7.3 \mathrm{~mm}$, since the operational current of the main coils was further decreased to $450 \mathrm{~A}$ in the meantime and additional tracking simulations indicated that this is the optimal shift to compensate for the integrated third order along the beam reference trajectory [14]. In all the measured wigglers a second order polynomial was sufficient to fit the exit angle as a function of the offset at the entrance, as shown in Fig. 8.

This implies that the maximum order detectable using this kind of analysis is the sextupole (which is of the same

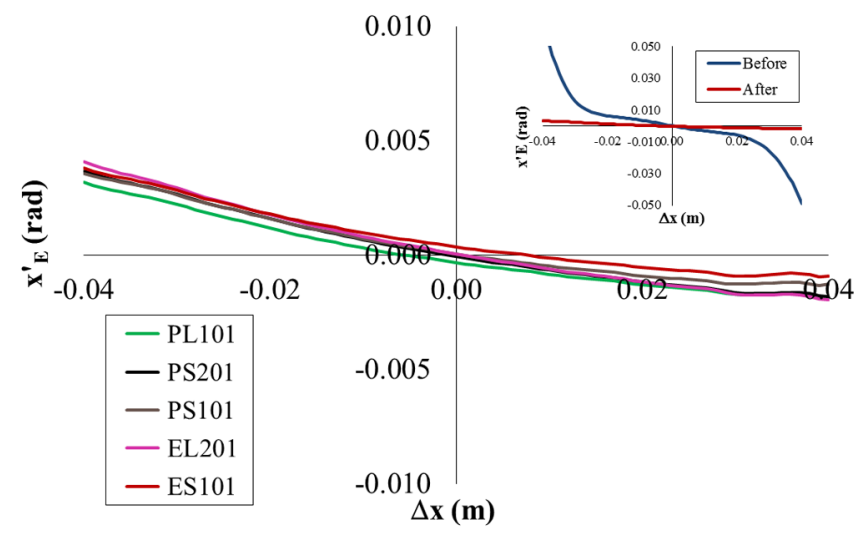

FIG. 8. Exit angle as a function of the orbit displacement at the entrance of the wiggler computed using the measured field maps after the modification. In the small graph the comparison with the aligned poles case (Before) is also shown. The results correspond to the first measured wigglers of the positrons (PL101, PS201, PS101) and electrons (EL201, ES101) ring. polarity of the second order introduced in the past to improve the dynamic aperture [11]). This indicates a complete compensation of the integrated third order term with respect to the beam reference trajectory.

\section{The beam based measurements}

After the first wigglers in the rings were modified tune shift measurements as a function of the amplitude of closed orbit local bumps $\Delta x$ localized at each wiggler position were performed to experimentally verify the method with beam. This type of measurement is one of the most accurate to quantify the nonlinearities, because in a ring the beam typically passes at least several millions times per second in the magnetic field, allowing even very small nonlinearities to be detected. The closed orbit bumps around the wiggler were generated in DA $\Phi N E$ by two correctors upstream and two steerers downstream the wiggler without any other active element in between to simultaneously control the position and the angle in the device. The strength of the steerers was calculated from the optics model and the ring lengthening induced by the bump was corrected by adjusting the rf frequency [14].

The tune shift, $\Delta Q_{x}$, up to the third order, as a function of the bump amplitude $\Delta x$ is given by

$$
\Delta Q_{x}=K_{2}^{\mathrm{MAD}} \cdot \frac{\beta_{x}}{4 \pi} \cdot \Delta x+K_{3}^{\mathrm{MAD}} \cdot \frac{\beta_{x}}{8 \pi} \cdot \Delta x^{2},
$$

where $\beta_{x}$ is the betatron oscillation function (a constant $\beta_{x}$ of $7 \mathrm{~m}$ in the wiggler was considered) and the terms $K_{k}^{\mathrm{MAD}}$ are defined in the MAD [15] notations as

$$
K_{i}^{\mathrm{MAD}} \equiv \int \frac{1}{B \rho} \frac{\partial^{i} B_{y}}{\partial x^{i}} d s .
$$

Each of the $K_{n}^{\mathrm{MAD}}$ is proportional to the $a_{k}$ terms in Eq. (6). In particular the $a_{2}$ term of Eq. (6), which corresponds to the integrated sextupole, is proportional to the slope of the tune shift as a function of $\Delta x$. The compensation of the integrated octupole with respect to the beam trajectory is demonstrated by the cancellation of the quadratic term in Eq. (8), where this term is proportional to the $a_{3}$ coefficient in Eq. (6) and therefore to $I_{3}$.

The tune shift measured in DA $\Phi N E$ is shown in Fig. 9.

The linear dependence confirms the suppression of the integrated octupole, and therefore experimentally proves the validity of the method. Furthermore for the case corresponding to the shifted poles configuration, the measurement is in agreement with the expectation, calculated from the coefficient $a_{2}$ obtained from the tracking studies applied to the measured field maps.

Following this, other measurements were performed in all the wigglers. The range of the bump amplitude has been further increased by up to $\pm 10.5 \mathrm{~mm}$ after the improvement of the water cooling of the correctors used for the 


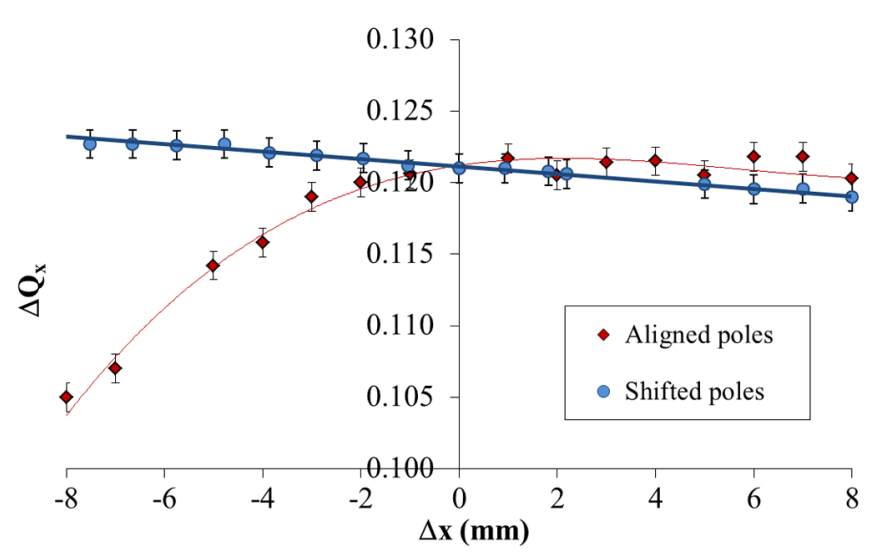

FIG. 9. Measured tune shift as a function of the closed orbit bump amplitude before (aligned poles) and after (shifted poles) the modification. For the case after the modification the line shows the predictions from the tracking analysis, for the case before the modification a polynomial fit to the experimental data is applied.

bumps. The measured integrated octupole was negligible also in this range [16].

\section{CONCLUSIONS}

Wiggler nonlinearities may strongly degrade the beam dynamics especially if the beam excursion is comparable to the pole width, as is typical for conventional wigglers. Several approaches have been tried in the past to mitigate this effect. The main author proposed an idea to cancel one specific order and reduce all the other odd integrated terms (the even being canceled by design in conventional devices for symmetry and periodicity conditions). This method was applied to the DA $\Phi$ NE main rings wigglers, where a large integrated octupole was being measured. The new configuration was optimized by multipolar studies and verified by tracking simulations.

All the wigglers in the DA $\Phi N E$ main rings were modified according to the optimal layout and the magnetic modeling was validated by $2 \mathrm{D}$ field map measurements. In this paper the experimental verification of this approach, which canceled the nonlinearities generated by the wigglers in DA $\Phi N E$ below the detectable level, was described.

The method is general and can be applied at zero cost to any periodic magnet in the design phase, allowing for an ideal magnet design from the field quality point of view and maximizing at the same time the field/current efficiency.

\section{ACKNOWLEDGMENTS}

We are grateful to the Frascati team for the experimental setup of the magnetic measurements, in particular to
Claudio Sanelli and Franco Iungo (Frascati INFN). We give a special thanks to Carlo Ligi (Frascati INFN) for prompt and effective help in fixing technical problems. We also thank Giovanni Bellomo (INFN Milan) and Davide Tommasini (CERN) for the fruitful discussions and Thomas Schietinger (PSI), M.E. Biagini (INFNFrascati), and Jan Chrin (PSI) for the revision of this paper.

[1] S. Guiducci et al., in Proceedings of the 2nd International Particle Accelerator Conference, San Sebastián, Spain (EPS-AG, Spain, 2011), MOOCA03.

[2] Y. Papaphilippou et al., in Proceedings of the $3 \mathrm{rd}$ International Particle Accelerator Conference, New Orleans, LA, 2012 (IEEE, Piscataway, NJ, 2012), TUPPC086.

[3] S. Guiducci et al., in Proceedings of the 9th European Particle Accelerator Conference, Lucerne, 2004 (EPS-AG, Lucerne, 2004), WEPKF034.

[4] J. Safranek, C. Limborg, A. Terebilo, K. Blomqvist, P. Elleaume, and Y. Nosochkov, Phys. Rev. ST Accel. Beams 5, 010701 (2002).

[5] S. Bettoni, Phys. Rev. ST Accel. Beams 10, 042401 (2007).

[6] C. Milardi et al., in Proceedings of the Particle Accelerator Conference, Chicago, IL, 2001 (IEEE, New York, 2001).

[7] A. Battisti et al., DA $\Phi$ NE Technical Note No. MM-34, 2004, TPPH027.

[8] S. Bettoni, S. Guiducci, M. Preger, P. Raimondi, and C. Sanelli, in Proceedings of the 22nd Particle Accelerator Conference, PAC-2007, Albuquerque, NM (IEEE, New York, 2007), THPE065.

[9] S. Bettoni et al., in Proceedings of the International Particle Accelerator Conference, Kyoto, Japan (ICR, Kyoto, 2010), THPE065.

[10] Opera 3D, Tosca, Software for Electromagnetic Design, Workstation, version 10.509, Size 3, Vector Fields Ltd., Oxford, England, Vector Fields Inc., Illinois, USA.

[11] C. Milardi et al., in Proceedings of the 23rd Particle Accelerator Conference, Vancouver, Canada, 2009 (IEEE, Piscataway, NJ, 2009), MO4RAI01.

[12] C. Milardi et al., "DA $\Phi$ NE developments for the KLOE-2 experimental Run," Proceedings of IPAC'10, Kyoto, Japan, 2010 TUPE006, p. 1527.

[13] S. Bettoni et al., DAథNE Technical Note No. MM-36, 2010.

[14] S. Bettoni, A. Drago, S. Guiducci, C. Milardi, M. Preger, and P. Raimondi, in Proceedings of the 2nd International Particle Accelerator Conference, San Sebastián, Spain, 2011, WEPC077.

[15] Methodical Accelerator Design code (MAD), http://cern .ch $/ \mathrm{mad}$.

[16] M. Preger (private communication). 